"This is the peer reviewed version of the following article: [ Digital literacy linked to engagement and psychological benefits among breast cancer survivors in Internet-based peer support groups. I

Lepore, Stephen J.; Rincon, Maria A.; Buzaglo, Joanne S.; Golant, Mitch; Lieberman, Morton

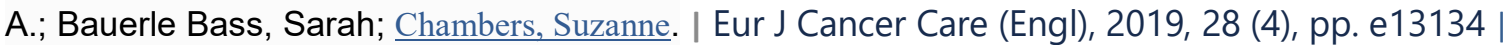

which has been published in final form at

[https://onlinelibrary.wiley.com/doi/abs/10.1111/ecc.13134]This article may be used for non-commercial purposes in accordance with Wiley Terms and Conditions for Self-Archiving 
Final accepted manuscript published as:

Lepore, S. J., Rincon, M., Buzaglo, J. S., Golant, M., Lieberman, M. A., \& Bass, S. B., (2019). Digital literacy linked to engagement and psychological benefits among breast cancer survivors in Internet-based peer support groups. European Journal of Cancer Care, 28, e13134. PMID: 31318132. DOI: 10.1111/ecc.13134

\section{Digital literacy linked to engagement and psychological benefits among breast cancer survivors in internet-based peer support groups}

Stephen J. Lepore, Temple University, slepore@temple.edu

Maria A. Rincon, Temple University

Joanne S. Buzaglo, The Cancer Support Community

Mitch Golant, The Cancer Support Community

Morton A. Lieberman, University of California, San Francisco

Sarah Bass, Temple University

Suzanne Chambers, University of Technology Sydney

Running title: Digital literacy and internet support groups

Keywords: Internet, breast neoplasms, social support, cancer survivors, literacy, psychological

Conflict of interest: The authors declare no conflict of interest.

Acknowledgements: This research was partially supported by grant R21CA15887 from the National Institutes of Health, National Cancer Institute. 


\begin{abstract}
Objective: Internet-based peer support groups (ISGs) represent an innovative, scalable approach to addressing information and support needs of cancer survivors. However, this innovation may not benefit survivors equally due to population variance in digital literacy. This study examined how digital literacy influences level of engagement in and psychological benefits from participating in ISGs for breast cancer $(N=183)$. Methods: Secondary analysis of data from a randomized trial of ISGs that included behavioral measures of engagement, subjective ratings, and psychological distress symptoms. Results: Digital literacy was positively related to education level $(p=.005)$. Relative to women with high digital literacy, those with lower digital literacy were more likely to report difficulties using the ISG and to value the user's guide and facilitator assistance (all p's<.05). Digital literacy was negatively correlated with computer anxiety preintervention, distress before and after online chat during the intervention, and postintervention depressive symptoms (all p's <.05). Conclusion: Low digital literacy is associated with computer anxiety and barriers to ISG use, as well as distress during and after ISG use. Digital literacy must be taken into account when designing or delivering innovative digital interventions for cancer survivors.
\end{abstract}




\section{Introduction}

Innovation in psycho-oncology will be largely driven by technology, particularly digitally-based interventions delivered via computers, cellphones, and other devices (Escriva Boulley et al., 2018). Digitally-based interventions are convenient to patients and efficient for delivering information and supportive care (Lee, Kim, \& Sharratt, 2018). Because cancer primarily afflicts older adults, the use of digital interventions in past years was impractical due to age-related access barriers. However, technology access has soared in recent years among older adults. For example, in early 2000, an estimated $14 \%$ of seniors were internet users, compared to $67 \%$ in 2017 (Anderson \& Perin, 2017). Now that this gap is narrowing, it is compelling to consider widespread applications of innovative, digitally-based interventions to address the psychosocial needs of cancer patients. However, in doing so, we need to be cognizant of other factors that might limit the efficacy of digitally-based interventions, including consumer usability and adoption. In this article, we investigate how breast cancer survivors' digital literacy affects how much they engage with and benefit from a popular digitally-based intervention: Internet support groups (ISGs) for cancer. The study aims to inform us about who might benefit most and least from such interventions and suggest ways to optimize the usability and efficacy of such interventions.

Breast cancer is among the most commonly diagnosed carcinomas in women, with over 260,000 estimated new cases in the United States alone in 2018 (American Cancer Society, 2018). The high incidence and decreasing mortality rates in breast cancer patients contributes to a growing number of survivors with long-term supportive care needs (American Cancer Society, 2018; Torre, Siegel, Ward, \& Jemal, 2016). It 
has been estimated that $32 \%-52 \%$ of women with breast cancer experience significant psychological distress at some stage of their diagnosis and treatment (Mehnert et al., 2018; Zabora, BrintzenhofeSzoc, Curbow, Hooker, \& Piantadosi, 2001). Using traditional, face-to-face psychological treatments to address the needs of so many patients would be cost prohibitive and unacceptable or impractical for many patients.

Digitally-based interventions could provide innovative, scalable means to address unmet psychosocial care needs in many cancer survivors (Fallon, Driscoll, Smith, Richardson, \& Portier, 2018). Indeed, just the number of ISGs for cancer has been estimated to exceed 400,000 (Im, Chee, Tsai, Lin, \& Cheng, 2005). Breast cancer ISGs are among the most active disease-specific support forums (Davison, Pennebaker, \& Dickerson, 2000). However, many patients may lack the knowledge or confidence to use their digital devices as a portal to psychosocial care. A national study of cancer survivors aged $65+$ found the vast majority rarely or never used the internet for health reasons (Lee et al., 2018). Among prostate and colorectal cancer survivors participating in a physical activity intervention, older age and lower educational level predicted a preference for print-based over Web-based intervention materials (Golsteijn et al., 2017).

Digital literacy may be a barrier to accessing and effectively using digitally-based interventions, such as cancer ISGs. Essentially, digital literacy is knowing how technology and digital media are used to communicate with others and gain knowledge and understanding (Hague \& Payton, 2010). When applied to the context of health, the term eHealth literacy is also used (Norman \& Skinner, 2006). Several population factors have been linked to lower digital literacy in a health context, including older age, lower 
educational level, and being a member of an ethno-racial minority group (Chesser, Burke, Reyes, \& Rohrberg, 2016). Although digital literacy is a relatively new concept in psycho-oncology, similar findings have begun to emerge in this area. For example, in a survey of lung cancer survivors, self-perceived digital literacy was generally low and inversely related to age (Milne et al., 2015).

To some extent, sociodemographic factors intersect and may influence digital literacy due to lower access to and experience in using technology (Chesser et al., 2016). One systematic review found lower educational status and income were reliably related to lower access to internet and communication technology (Fang et al., 2018). Home broadband access, which is critical for efficiently browsing the internet and downloading or streaming multimedia, also varies by socioeconomic status and race: $87 \%$ of families earning $\geq \$ 75,000$ per year have home broadband vs. $45 \%$ of those earning $\leq \$ 30,000$ per year; $72 \%$ of white vs. $57 \%$ black adults have home broadband; and $85 \%$ of college vs. $56 \%$ of high school graduates have home broadband (Pew Research Center, 2018). Lower access to computers and reliable, high-speed connectivity can translate to lower computer experience, which is linked to poorer computer task performance and learning outcomes in older adults (Xie, 2011).

As innovative and promising as they seem, we know relatively little about the broad acceptability and efficacy of digital interventions in cancer survivors because of the great population heterogeneity and lack of adequate controls across studies (Escriva Boulley et al., 2018). In one systematic review of ISGs and resources for cancer survivors, only four out of 24 studies used a gold standard randomized controlled trial design (Hong, Peña-Purcell, \& Ory, 2012). A more recent review of six 
randomized trials of ISGs for breast cancer survivors concluded that there was insufficient evidence on the efficacy of the interventions because the trials were generally low quality (McCaughan, Parahoo, Hueter, Northouse, \& Bradbury, 2017). We know little about how digital literacy influences the uptake and efficacy of digital interventions, including cancer ISGs, because literacy is seldom addressed in digital health interventions (Welch, Petkovic, Pardo Pardo, Rader, \& Tugwell, 2016). However, there is reason to believe that it may be challenging to deliver effective digital interventions to ethnically and economically diverse populations due to low digital literacy. One recent study with cancer survivors also found a less positive attitude toward digital interventions among those older in age and lower in education and income (Jansen, van Uden-Kraan, van Zwieten, Witte, \& Verdonck-de Leeuw, 2015).

To address gaps in our understanding of the significance of digital literacy to digital interventions in psychosocial cancer care, the current study examined demographic predictors of digital literacy, as well as the relation of digital literacy to level of intervention engagement and psychological outcomes in women participating in ISGs for breast cancer. Through secondary analyses, we were able to address the following questions:

1. Are breast cancer survivors participating in ISGs at risk for lower digital literacy if they are older or have lower levels of formal education? Based on the literature (Tennant et al., 2015), we hypothesized:

a. Digital literacy would be higher among participants with more formal education than among those with relatively less formal education.

b. Digital literacy would be higher among younger than older participants. 
2. To inform future intervention development, we asked whether the availability of an ISG professional facilitator and a printed ISG user guide were perceived to be particularly helpful among participants with lower digital literacy. We hypothesized:

a. Helpfulness of the ISG user guide would be rated higher among participants with low digital literacy than among those with relatively high digital literacy. b. Helpfulness of the ISG facilitators would be rated higher among participants with low digital literacy than among those with relatively high digital literacy. 3. By definition, low digital literacy makes it difficult for individuals to use computers and computer applications (Norman \& Skinner, 2006; Watkins \& Xie, 2014). Research has shown that higher digital literacy is linked to adoption of new technology (Hasan \& Ahmed, 2010; Potosky, 2007). Hence, we were interested in examining whether low digital literacy created barriers to using and fully engaging in the ISGs. We hypothesized:

a. Perceived difficulties in using the discussion board would be more frequent among participants with low digital literacy than those with relatively high digital literacy.

b. The experience of computer problems interfering with ISG participation would be more frequent among participants with low digital literacy than those with relatively high digital literacy.

c. Amount of engagement in the chat room (words communicated) would be lower among participants with low digital literacy than those with relatively high digital literacy. 
4. Finally, a central goal of this investigation was to better understand the extent to which digital literacy affects psychological well-being before, during and after participating in a cancer ISG. We hypothesized:

a. Digital literacy would be inversely related to computer anxiety prior to intervention (MacCallum, Jeffrey, \& Kinshuk, 2014).

b. Digital literacy would be inversely related to distress levels while using the ISG (i.e., distress before and after using chat).

c. Digital literacy would be inversely related to post-intervention depression symptoms, controlling or pre-intervention symptoms and other covariates. d. Digital literacy would be inversely related to post-intervention anxiety symptoms, controlling or pre-intervention symptoms and other covariates.

Methods

Study population and overview

We conducted secondary analyses of data collected between 2011-2012 from a randomized controlled trial investigating the efficacy of ISGs on psychological distress in breast cancer survivors (for details, see: Lepore et al., 2014; Lepore, Buzaglo, Lieberman, Golant, \& Davey, 2011). Theoretically, members of cancer ISGs benefit from receiving social support and, according to the helper therapy principle, by providing support to others (Post, 2007; Reissman, 1965). The randomized trial tested the psychological benefits of providing support to others by comparing the efficacy of a standard ISG (S-ISG) focused on seeking and receiving support and an enhanced prosocial ISG (P-ISG) that encouraged provision of support to others in addition to seeking and receiving support. 
The two-armed trial with 1-month pre- and post-intervention assessments was conducted with women who had been diagnosed with non-metastatic breast cancer and reported elevated symptoms of anxiety or depression. Women were recruited via a State Cancer Tumor registry. Eligibility criteria included: treated for stage I or II breast cancer in the past 36 months; age 21 to 65 years; Internet access; fluency in English; and elevated distress (scoring above normal [> 8] for levels of depression or anxiety on the Hospital Anxiety and Depression Scale) (Zigmond \& Snaith, 1983). Temple University's Institutional Review Board approved the protocol. Women were screened for eligibility and consented via telephone. Following enrollment, women completed a structured telephone interview to provide pre-intervention data. They were then randomized to either the S-ISG or P-ISG condition. Post-intervention survey data were collected within one month after the intervention via telephone to assess the primary outcomes and participants' assessment of the intervention.

Twelve ISGs were run (six per condition, with a median of 15 members per group). All groups received weekly, 90-minute professionally facilitated synchronous (participating together, in real time) chat sessions for six weeks (90-minute weekly sessions) and 24/7 access to an asynchronous (individuals can participate at any time) discussion board. Chat sessions were facilitated by trained and clinically supervised Master's-level professionals and structured around prearranged topics (e.g., managing symptoms). All participants received an illustrated user's guide explaining the ISG features and functions and a handout of tips on how to get support in an ISG. The PISG condition included additional tips on how to give support in an ISG and had structured opportunities and encouragement to help others. Prior to the first chat 
session, participants were instructed to post a test message on the discussion board. Anyone who did not log on within 24 hours was contacted by phone and further guided through the process if necessary. The chat room used varied text colors to differentiate participants on the screen and included a set of emojis to facilitate communication. Participants had access to the transcripts of the chat sessions within 24 hours.

Discussion board threads could be about any topic. Measures

Demographic and clinical characteristics: In the pre-intervention telephone survey, we assessed demographics (age, race, education, employment status, marital status) and verified clinical data collected from the tumor registry (stage of cancer, months since diagnosis, and type of primary treatment).

Digital literacy: In order for cancer survivors to effectively access and engage with the study ISG, they need to be proficient in navigating the internet to find information, using communication tools (email, chat, messaging), interacting with documents (downloading transcripts), and discerning credible information. Potosky (2007) developed two scales to measure internet knowledge and skills related to information searching and communication. These scales captured aspects of digital literacy we deemed relevant to using cancer ISGs: navigation skill (e.g., "When using the internet, I quickly find information that I am looking for"); communication skill (e.g., "I use the internet/email to communicate with other people"); discern credible information (e.g., "I can usually recognize and avoid spam e-mail messages"); and basic functions (e.g., "I know how to upload and send attachments with e-mail"). In the pre-intervention telephone survey, we used 10 of the original 11 items developed by Potosky and added 
two health-specific items (e.g., "I have searched the internet for breast cancer information," "I have searched the internet for health information"). Respondents rated each of the 12 items on a 4 -point scale $(1=$ strongly disagree to $4=$ strongly agree $)$. Analyses of the original subscales revealed them to be very highly and positively correlated, $r(183)=.73, p=.000$, so we combined all items into a composite scale. Items were averaged, with higher scores indicative of higher digital literacy. This face valid measure had strong reliability (Cronbach's alpha $=.91)$.

Helpfulness of ISG aids: In the post-intervention telephone survey, participants rated the helpfulness $(1=$ not at all to $5=$ very much $)$ of the ISG aids: (a) 'the user's guide for learning how to use the study website' and (b) 'the group facilitator'. Higher scores indicate greater perceived helpfulness of the ISG aids.

Perceived barriers to ISG use: In the post-intervention telephone survey, participants rated the extent $(1=$ not at all to $5=$ very much) to which they experienced two barriers to ISG use: (a) 'computer problems made it difficult to use the study website to participate in the ISG' and (b) 'difficulty using the discussion board on the study website'. Higher scores indicate greater perceived barriers.

Engagement in ISG. Using the Linguistic Inquiry and Word Count (Pennebaker, Booth, Boyd, \& Francis, 2015) software program, we were able to analyze transcripts of the six weekly synchronous chat sessions to count the number of words posted by each participant. A higher number of words indicates greater engagement.

Distress: Various distress indicators were measured before, during and after the intervention. In the pre-intervention telephone survey, computer anxiety was measured 
with a four-item scale (Barbeite \& Weiss, 2004). Participants rated how much they agreed $(1=$ strongly disagree to $4=$ strongly agree) with four statements about computer anxiety (e.g., working with a computer would make me nervous). Scores were averaged, with higher scores indicating greater computer anxiety. The measure had strong reliability (Cronbach's alpha $=.91)$. During the six weekly chat sessions, participants rated distress using an 11-point, interactive distress thermometer $(0=$ no distress to $10=$ extreme distress) that appeared on the computer screen whenever a participant entered or exited the chat room. With a mouse or track pad, participants could move the "mercury" in the digital analogue thermometer to indicate their current level of distress. Pre- and post-chat distress scores were calculated by averaging across chat sessions, with higher scores indicating greater distress. In the pre- and post-intervention telephone surveys, the Hospital Anxiety and Depression Scale was used to measure psychological symptomatology (Zigmond \& Snaith, 1983). The measure generates depression symptoms and anxiety symptoms subscales and has been validated with breast cancer populations (Jacobsen et al., 2005). Higher scores indicate greater symptoms. The measures had good reliability (Cronbach's alpha for both scales $=.83$ ).

Analytic approach

Attrition was low in the study, with $87 \%$ of randomized participants completing the post-intervention assessment. Pre-intervention variables in Table 1 did not differ significantly between participants who completed the post-intervention assessment $(n=$ $160)$ and those who were lost to follow-up $(n=23)$. We used pairwise deletion of cases in analyses with missing data due to attrition or non-response and set the level of 
significance for statistical tests at .05 . Results from the parent study showed that psychological symptoms improved in both experimental conditions from pre-intervention to post-intervention, but contrary to expectations participants in the S-ISG condition had significantly lower post-intervention symptoms of anxiety and depression than participants in the P-ISG condition (Lepore et al., 2014). Thus, for analyses examining post-intervention symptoms, intervention condition was one of the statistical control variables. In analyses predicting post-intervention distress symptoms, we also statistically controlled for pre-intervention psychological symptoms, age, and level of education to rule out potential confounded relations between these variables.

Analyses of the relation between digital literacy and categorical factors (e.g., education level) used independent sample t-tests, whereas analyses between digital literacy and continuous factors (e.g., age, computer anxiety) used zero-order Pearson's correlations. In addressing the primary study question about the potential influence of digital literacy on psychological benefits of participating in an ISG, we performed separate multiple linear regression analyses on depression and anxiety symptom scores. To rule out potential confounders, age, education level, and experimental condition were statistically controlled in the regression analyses. In addition, the corresponding pre-intervention symptom subscale score was statistically controlled in each regression analysis (e.g., pre-intervention depression when post-intervention depression was the outcome).

\section{Results}

Table 1 shows the pre-intervention characteristics of the sample. Participants, who ranged in age from 29 to 65, were mostly Caucasian, highly educated, employed, 
and married. More participants had stage 1 than stage 2 disease, the majority were more than 18-months postdiagnosis, and surgery was the most common primary treatment. Participants attended most of the weekly chat sessions (Md $=5$ weeks out of 6) but used the discussion board relatively little ( $M d=2$ weeks out of 6$)$ (not shown in Table 1).

Table 2 shows descriptive data on the major study variables. On average, participants reported moderately high digital literacy, wrote a good deal in the chat sessions, experienced few computer problems or difficulties using the discussion board, and found the user guide to be moderately helpful and the ISG facilitator more so. On average, pre-intervention computer anxiety was low. Average level of distress before entering the chat room was low and significantly lower by the end of the chat session. Symptoms of anxiety and depression pre-intervention were moderately high, on average, and significantly lower by post-intervention.

The first set of hypotheses focused on predictors of digital literacy. As predicted, digital literacy was higher among college graduates $(M=3.59, \mathrm{SD}=.40)$ than noncollege graduates $(M=3.41, \mathrm{SD}=.46), t(181)=2.82, p=.005$. Further, digital literacy was inversely correlated with age, but not significantly so, $r(183)=-.12, p=.10$.

The second set of hypotheses focused on the relation between digital literacy and perceived helpfulness of the ISG aids. As predicted, digital literacy was negatively correlated with the perceived helpfulness of the user's guide, $r(145)=-.21, p=.011$ and the online group facilitator, $r(146)=-.18, p=.027$.

The third set of hypotheses focused on the relation between digital literacy and perceived barriers to using the ISG and level of engagement in using the ISG. As 
predicted, digital literacy was negatively correlated with perceived difficulty using the discussion board, $r(146)=-.36, p=.000$, and the perception that computer problems interfered with ISG participation, $r(147)=-.17, p=.043$. Consistent with the engagement hypothesis, digital literacy was positively correlated with total words expressed during live chat, $r(183)=.15, p=.037$.

The final set of hypotheses focused on the relation between digital literacy and psychological distress, including anxiety about using computers in general, distress while using a cancer ISG, and psychological distress symptoms (depression/anxiety) at completion of a cancer ISG. As predicted, digital literacy was inversely correlated with level of computer anxiety pre-intervention, $r(183)=-.67, p=.000$, and average level of negative mood expressed on the mood thermometer prior to entering chat, $r(161)=-$ $.20, p=.000$, and immediately after exiting chat, $r(161)=-.29, p=.000$.

Results of the regression analyses on psychological outcomes were largely consistent with hypotheses. A significant overall regression equation was found for postintervention depression, $F(5,154)=29.75, p=.000$, with an $R^{2}$ of .70 . Digital literacy was related to significantly lower post-intervention depressive symptoms, independent of pre-intervention depressive symptoms, experimental condition, age, and education level, $\mathrm{B}=-1.20, \mathrm{SE}=.60, B=-.12, t=-2.00, p=.047$, accounting for $1.3 \%$ of the variance. A significant overall regression equation also was found for post-intervention anxiety, $\mathrm{F}(5,154)=14.32, p=.000$, with an $R^{2}$ of .56 . Digital literacy was related to lower post-intervention anxiety symptoms independent of control variables, but not significantly so, $\mathrm{B}=-1.23, \mathrm{SE}=.68, B=-.12, t=-1.80, p=.074$. 
This study showed that digital literacy was related in an expected fashion to sociodemographic factors and psychological and behavioral outcomes in women using ISGs for breast cancer. Lower digital literacy was linked to lower education and older age, although the relation to age was not statistically significant. Relative to women high in digital literacy, women with relatively low digital literacy reported more problems using their computer to access the ISG, more difficulties using the discussion board, and they did not express themselves as much in the ISG chat rooms. Lower digital literacy also was associated with higher computer anxiety pre-intervention, higher distress before and after the ISG chat sessions, and higher depressive symptoms post intervention. To our knowledge, this is the first study to link low digital literacy to poorer engagement in an ISG and to poorer psychological outcomes before, during and after using a cancer ISG designed to improve psychological outcomes.

Our criteria for patient selection into the parent study truncated the upper age range, which likely accounts for the observed non-significant negative correlation between age and digital literacy. In addition, the sample was mostly highly educated, which may have further restricted the variance in digital literacy and attenuated the size of the observed association between education and digital literacy. The challenges in recruiting and retaining women from socially disadvantaged groups in breast cancer survivorship research are well known. Proactive, in-person recruitment approaches (Yancey, Ortega, \& Kumanyika, 2006), community-based approaches in which investigators partner with local organizations and community leaders (Horowitz, Brenner, Lachapelle, Amara, \& Arniella, 2009), and financial incentives (Satia, Galanko, \& Rimer, 2005) may improve recruitment of individuals from lower socioeconomic and 
ethnoracial minority groups into future research studies on digital literacy and cancer survivorship.

This study clearly showed that despite access to the internet via personal computers and laptops, a subset of women still faced difficulties in effectively interacting with the ISG. For example, lower digital literacy was associated with more problems using the study website, which may have accounted for some of the lower levels of observed participation in the chat room sessions. These barriers, along with general computer anxiety, also may have contributed to elevations in distress levels during chat room sessions evident among women with lower digital literacy. Further, while the average study participant experienced improvements in psychological distress symptoms from pre-intervention to post-intervention, those with lower digital literacy tended to have more post-intervention distress than their counterparts with high digital literacy. The data linking digital literacy to depression symptoms are correlational, but did adjust for pre-intervention symptoms, experimental condition, age, and education level. These findings suggest a possibility that cancer survivors with low digital literacy may not benefit as much from digital interventions as their counterparts with higher digital literacy.

It is perhaps encouraging that women with lower digital literacy were willing to try the ISG intervention despite their trepidations. In addition, in comparison with women high in digital literacy, women with relatively low digital literacy expressed greater appreciation of the provided technical guides and assistance. These kinds of supports, and possibly other forms of tutorials or trainings, could improve digital literacy and make digital interventions more accessible to older and socially disadvantaged cancer 
populations. Patient education and training around the use of digital technology could be offered in cancer centers or community-based technology centers (Salovey et al., 2009). Of course, it will not be a trivial matter to provide training to a clinical population that may be fatigued, in pain, or unable to travel. In addition to training, using available toolkits to design maximally accessible websites (U.S. Department of Health and Human Services, 2015) is critical for making interventions inclusive for persons with low digital literacy. Interventions also could be tailored to the level of digital literacy of target populations (Watkins \& Xie, 2014), just as we should tailor them to level of general literacy. Finally, it may be fruitful to enlist peers and family members to help patients take advantage of digital interventions. Rather than directly searching for information on the internet or communicating through e-mail, many persons with cancer do so indirectly through family and friends (Eysenbach, 2003).

\section{Limitations}

One limitation of this study is the use of a self-report measure of digital literacy. Any such measure will capture some but not all of the domains of the digital literacy construct and will depend on respondents' level of self-awareness about their digital knowledge and skills. Observing individuals as they use a digital intervention and respond to questions and challenges related to different features of the intervention would likely reveal a more accurate picture of digital knowledge and skills. Nonetheless, as the current findings reveal, a self-report measure is sufficient for identifying individuals who may be apprehensive and less prepared to engage with and benefit from a digital intervention. It is important when selecting a measure to ensure that the 
items reflect the kind of task demands used in the intervention and that it is not outdated.

Another limitation is the unrepresentative sample of breast cancer survivors. We do not know the extent to which these results would generalize to older or more socially disadvantaged populations, but in all likelihood, we would see more problems with digital literacy and stronger associations with outcomes than reported herein. Implications

The findings suggest that low digital literacy among cancer survivors may impede the degree to which they use and benefit from ISGs, a common source of support and information on the internet. As digital resources continue to grow and become a significant source of information and support offered by clinics, government agencies, and non-profit organizations serving cancer survivors, we may be creating a large population of people who are disadvantaged due to low digital literacy. These findings are a caution against the overuse of digital interventions in the psychosocial care of cancer survivors, particularly patients who are older, have relatively low education and computer experience, and live in areas with inadequate internet access (Chesser et al., 2016). Comprehensive approaches that include traditional support services and formats are still warranted. Simultaneously, we should seek to apply best practices (U.S. Department of Health and Human Services, 2015) to design digital interventions that are accessible to individuals with limited literacy skills and Web experience, and attend to the digital training and technical support needs of patients. In the present study, women with lower digital literacy reported challenges and distress when using the ISGs, but they also felt that the user's guide and facilitator were helpful to them and they 
persisted in using the ISG despite these difficulties. This suggests that the perceived positives of connecting with other patients via the ISG outweighed the negatives for women with breast cancer and motivated them to cross the digital divide. 


\section{References}

American Cancer Society. (2018). Cancer Facts \& Figures 2018. Retrieved April 29, 2018, from https://www.cancer.org/research/cancer-facts-statistics/all-cancer-factsfigures/cancer-facts-figures-2018.html

Anderson, M., \& Perin, A. (2017). Tech adoption climbs among older adults. Retrieved April 29, 2018, from https://www.pewinternet.org/2017/05/17/tech-adoption-climbsamong-older-adults/

Barbeite, F. G., \& Weiss, E. M. (2004). Computer self-efficacy and anxiety scales for an Internet sample: testing measurement equivalence of existing measures and development of new scales. Computers in Human Behavior, 20(1), 1-15.

Chesser, A., Burke, A., Reyes, J., \& Rohrberg, T. (2016). Navigating the digital divide: A systematic review of eHealth literacy in underserved populations in the United States. Informatics for Health and Social Care, 41(1), 1-19.

Davison, K. P., Pennebaker, J. W., \& Dickerson, S. S. (2000). Who talks? The social psychology of illness support groups. American Psychologist, 55(2), 205-217.

Escriva Boulley, G., Leroy, T., Bernetière, C., Paquienseguy, F., Desfriches-Doria, O., \& Préau, M. (2018). Digital health interventions to help living with cancer: A systematic review of participants' engagement and psychosocial effects. PsychoOncology.

Eysenbach, G. (2003). The impact of the internet on cancer outcomes. CA: A Cancer Journal for Clinicians, 53(6), 356-371.

Fallon, E. A., Driscoll, D., Smith, T. S., Richardson, K., \& Portier, K. (2018). Description, characterization, and evaluation of an online social networking community: the 


\section{American Cancer Society's Cancer Survivors Network. Journal of Cancer}

Survivorship, 12(5), 691-701.

Fang, M. L., Canham, S. L., Battersby, L., Sixsmith, J., Wada, M., \& Sixsmith, A. (2018).

Exploring Privilege in the Digital Divide: Implications for Theory, Policy, and

Practice. The Gerontologist. https://doi.org/10.1093/geront/gny037

Golsteijn, R. H. J., Bolman, C., Peels, D. A., Volders, E., de Vries, H., \& Lechner, L. (2017). A Web-based and print-based computer-tailored physical activity intervention for prostate and colorectal cancer survivors: a comparison of user characteristics and intervention use. Journal of Medical Internet Research, 19(8), e298.

Hague, C., \& Payton, S. (2010). Digital literacy across the curriculum: a Futurelab handbook. Retrieved March 11, 2018, from https://www.nfer.ac.uk/publications/FUTL06/FUTL06.pdf

Hasan, B., \& Ahmed, M. U. (2010). A path analysis of the impact of application-specific perceptions of computer self-efficacy and anxiety on technology acceptance. Journal of Organizational and End User Computing, 22(3), 82-95.

Hong, Y., Peña-Purcell, N. C., \& Ory, M. G. (2012). Outcomes of online support and resources for cancer survivors: a systematic literature review. Patient Education and Counseling, 86(3), 288-296.

Horowitz, C. R., Brenner, B. L., Lachapelle, S., Amara, D. A., \& Arniella, G. (2009). Effective recruitment of minority populations through community-led strategies. American Journal of Preventive Medicine, 37(6), S195-S200.

Im, E.-O., Chee, W., Tsai, H.-M., Lin, L.-C., \& Cheng, C.-Y. (2005). Internet cancer 
support groups: a feminist analysis. Cancer Nursing, 28(1), 1-7.

Jacobsen, P. B., Donovan, K. A., Trask, P. C., Fleishman, S. B., Zabora, J., Baker, F., \& Holland, J. C. (2005). Screening for psychologic distress in ambulatory cancer patients. Cancer, 103(7), 1494-1502.

Jansen, F., van Uden-Kraan, C. F., van Zwieten, V., Witte, B. I., \& Verdonck-de Leeuw, I. M. (2015). Cancer survivors' perceived need for supportive care and their attitude towards self-management and eHealth. Supportive Care in Cancer, 23(6), 16791688.

Lee, H. Y., Kim, J., \& Sharratt, M. (2018). Technology use and its association with health and depressive symptoms in older cancer survivors. Quality of Life Research, 27(2), 467-477.

Lepore, S. J., Buzaglo, J. S., Lieberman, M. A., Golant, M., Greener, J. R., \& Davey, A. (2014). Comparing standard versus prosocial internet support groups for patients with breast cancer: a randomized controlled trial of the helper therapy principle. Journal of Clinical Oncology, 32(36), 4081-4086.

Lepore, S. J., Buzaglo, J. S., Lieberman, M. a, Golant, M., \& Davey, A. (2011). Standard versus prosocial online support groups for distressed breast cancer survivors: a randomized controlled trial. BMC Cancer, 11(1), 379.

MacCallum, K., Jeffrey, L., \& Kinshuk. (2014). Factors impacting teachers' adoption of mobile learning. Journal of Information Technology Education, 13, 141-162.

McCaughan, E., Parahoo, K., Hueter, I., Northouse, L., \& Bradbury, I. (2017). Online support groups for women with breast cancer. Cochrane Database of Systematic Reviews, 3, CD011652. 
Mehnert, A., Hartung, T. J., Friedrich, M., Vehling, S., Brähler, E., Härter, M., ... Faller, H. (2018). One in two cancer patients is significantly distressed: Prevalence and indicators of distress. Psycho-Oncology, 27(1), 75-82.

Milne, R. A., Puts, M. T. E., Papadakos, J., Le, L. W., Milne, V. C., Hope, A. J., ... Giuliani, M. E. (2015). Predictors of High eHealth Literacy in Primary Lung Cancer Survivors. Journal of Cancer Education, 30(4), 685-692.

Norman, C. D., \& Skinner, H. A. (2006). eHealth literacy: essential skills for consumer health in a networked world. Journal of Medical Internet Research, 8(2), e9.

Pennebaker, J. W., Booth, R. J., Boyd, R. L., \& Francis, M. E. (2015). Linguistic Inquiry and Word Count: LIWC2015 Operator's Manual. Austin, TX: Pennebaker Conglomerates.

Pew Research Center. (2018). Internet/broadband fact sheet. Retrieved April 11, 2018, from http://www.pewinternet.org/fact-sheet/internet-broadband/

Post, S. G. (2007). Altruism and health: Perspectives from empirical research. New York, NY: Oxford.

Potosky, D. (2007). The Internet knowledge (iKnow) measure. Computers in Human Behavior, 23(6), 2760-2777.

Reissman, F. (1965). The helper-therapy principle. Social Work, 10, 27-32.

Salovey, P., Williams-Piehota, P., Mowad, L., Moret, M. E., Edlund, D., \& Andersen, J. (2009). Bridging the digital divide by increasing computer and cancer literacy: community technology centers for head-start parents and families. Journal of Health Communication, 14(3), 228-245.

Satia, J. A., Galanko, J. A., \& Rimer, B. K. (2005). Methods and strategies to recruit 
African Americans into cancer prevention surveillance studies. Cancer Epidemiology Biomarkers \& Prevention, 14(3), 718-721.

Tennant, B., Stellefson, M., Dodd, V., Chaney, B., Chaney, D., Paige, S., \& Alber, J. (2015). eHealth literacy and Web 2.0 health information seeking behaviors among baby boomers and older adults. Journal of Medical Internet Research, 17(3), e70.

Torre, L. A., Siegel, R. L., Ward, E. M., \& Jemal, A. (2016). Global cancer incidence and mortality rates and trends-an update. Cancer Epidemiology Biomarkers \& Prevention, 25(1), 16-27.

U.S. Department of Health and Human Services. (2015). Health literacy online: 2nd Edition. Retrieved November 26, 2018, from https://health.gov/healthliteracyonline/

Watkins, I., \& Xie, B. (2014). eHealth literacy interventions for older adults: a systematic review of the literature. Journal of Medical Internet Research, 16(11), e225.

Welch, V., Petkovic, J., Pardo Pardo, J., Rader, T., \& Tugwell, P. (2016). Interactive social media interventions to promote health equity: an overview of reviews. Health Promotion and Chronic Disease in Canada, 36, 63-75.

Xie, B. (2011). Effects of an eHealth literacy intervention for older adults. Journal of Medical Internet Research, 13(4), e90.

Yancey, A. K., Ortega, A. N., \& Kumanyika, S. K. (2006). Effective recruitment and retention of minority research partciipants. Annual Review of Public Health, 27(1), $1-28$.

Zabora, J., BrintzenhofeSzoc, K., Curbow, B., Hooker, C., \& Piantadosi, S. (2001). The prevalence of psychological distress by cancer site. Psycho-Oncology, 10(1), 1928. 
Digital literacy and internet support groups 26

Zigmond, A. S., \& Snaith, R. P. (1983). The hospital anxiety and depression scale. Acta Psychiatrica Scandinavica, 67(6), 361-370. 
Table 1. Pre-intervention demographic and clinical characteristics of sample $(N=$ 183).

\begin{tabular}{lcl}
\hline Variable & No. of \\
& Participants & $\%^{1}$ \\
\hline
\end{tabular}

Age, years

$29-51$

72

39.3

$52-65$

111

60.7

Race Caucasian

174

95.6

Educational attainment

High school graduate

31

16.9

Some college

62

33.9

College graduate

51

27.9

Post graduate degree

39

21.3

Employed part- or full-time

68.9

Married/in marital-like relation

156

85.2

Stage of cancer

Months since diagnosis

$$
\begin{array}{r}
6-18 \\
19-36
\end{array}
$$

135

73.8

Surgical treatment

153

95.6

Chemotherapy treatment

103

64.4

Radiotherapy treatment

67

41.9 
Table 2. Descriptive data on major study variables.

\begin{tabular}{|c|c|c|}
\hline Variable & Mean & SE \\
\hline Digital literacy pre-intervention ${ }^{1}$ & 3.49 & .03 \\
\hline Word expressed in chat sessions & 1967.72 & 116.20 \\
\hline Computer problems made it difficult to use ISG ${ }^{2}$ & 1.50 & .07 \\
\hline Difficulty using the discussion board & 0.32 & .06 \\
\hline Helpfulness of ISG user guide & 3.43 & .12 \\
\hline Helpfulness of professional ISG facilitator & 4.44 & .07 \\
\hline Computer anxiety pre-intervention & 1.33 & .04 \\
\hline \multicolumn{3}{|l|}{ Distress thermometer scores } \\
\hline Before entering chat sessions ${ }^{3}$ & 3.34 & .17 \\
\hline After entering chat sessions ${ }^{3}$ & $2.58^{*}$ & .16 \\
\hline \multicolumn{3}{|l|}{ Depressive symptoms } \\
\hline Pre-intervention ${ }^{4}$ & 6.93 & .28 \\
\hline Post-intervention & $5.94^{*}$ & .34 \\
\hline \multicolumn{3}{|l|}{ Anxiety symptoms } \\
\hline Pre-intervention & 10.39 & .23 \\
\hline Post-intervention & $8.44^{*}$ & .34 \\
\hline
\end{tabular}

Notes: ${ }^{1}$ Pre-intervention data were collected by structured telephone survey within 1 month prior to the intervention. Post-intervention data were collected by structured telephone survey within 1 month after the intervention. ${ }^{2} \mathrm{ISG}=$ Internet Support Group. ${ }^{3}$ Averaged across the six chat sessions. ${ }^{4}$ All symptom scores are raw, unadjusted means. ${ }^{*} p<.001$, paired t-test analysis of means pre- to postintervention. 